\title{
A Collaborative Planning, Information and Decision Support System
}

\author{
Sobah Abbas Petersen ${ }^{1}$, Pavan Sriram ${ }^{2}$, John Krogstie ${ }^{3}$, \\ Børge Sjøbakk ${ }^{1}$, and Ottar Bakås ${ }^{1}$ \\ ${ }^{1}$ SINTEF Technology and Society, Norway \\ \{Sobah. Petersen, Borge.Sjobakk, Ottar.Bakas\} @ sintef.no \\ ${ }^{2}$ Dept. of Production and Quality Engineering, NTNU, Norway \\ Pavan.sriramantnu.no \\ ${ }^{3}$ Dept. of Computer and Information Science, NTNU, Norway \\ Krogstie@idi.ntnu.no
}

\begin{abstract}
In this paper, we discuss the importance of collaborative planning for manufacturing enterprises. We have analysed user stories provided by industry to understand the collaborative processes in their workplaces and their needs for Information Systems (IS) support. Based on the analysis of the user stories, we have proposed a framework for collaborative planning and ideas for the design of IS supporting this activity. These will be used to conduct focussed interviews with the users to refine our design before implementing the system.
\end{abstract}

Keywords: Collaborative planning, Collaborative Processes, Global Manufacturing, Knowledge Management Systems, Decision Support, User Stories.

\section{Introduction}

Globalization has made manufacturers face increased challenges for better product quality and shorter delivery time [1]. To remain competitive in the market, organisations have to cope with pressure on prices, smaller orders, more suppliers, more governmental regulation and increasing material and energy costs. These new business drivers have made it necessary for manufacturing enterprises to collaborate with each other, their customers and suppliers to leverage each other's competences to remain competitive. This has increased the need for Information Systems (IS) support for collaborative planning across organizational borders. The behaviour, activities and all the dynamic aspects of the manufacturing enterprises highly depend on information systems and the processes, services, and data they manage [2].

Today's market is dynamic and uncertain. The products are highly customized and diverse in nature. The environment consists of repetitive (standard) and non-repetitive (customized) components and tasks. There is a high complexity in the nature of products, with short lead time, increased outsourcing and distributed teams who are trying to rapidly fulfill the needs of the customers. To effectively tackle these challenges and to obtain competitive advantage in today's internet economy, an efficient collaborative planning, information and decision support system is required. Collaborative 
practices and methodologies have been developed for coordination between long term partners. Examples include Vendor Inventory Management and Collaborative Planning, Forecasting and Replenishment [3]. More recently, a collaborative model, Coagmento, for collaborative information seeking has been developed [4].

There is a growing interest in understanding relationships and interactions both within and between organisations, how they collaborate and how IS can support this. The benefits of collaboration to alleviate, or ideally to eliminate, various problems and allowing the partners to pool resources and exploit complementarities are still far from being fully realised. Over the past few years, the researchers and practitioners have recognised the value derived via collaborative processes. However, frameworks, architectures and application that coherently integrate all the information and planning systems are scarce [5]. The analysis of user stories and the design of a framework for collaborative planning discussed in this paper aims to address this gap and provide a fresh perspective in this multi-disciplinary area of research. This is done in three stages: (i) obtaining user stories and analysing them to find the shortcomings of collaborative planning within and across organisations; (ii) exploring the literature and industrial applications of IS; and (iii) proposing the design of a system for collaborative planning, information and decision support. The data used for our study are from samples of user stories or statements obtained through interviews. In this paper, we propose design ideas for a framework for supporting collaborative planning. The design ideas and the proposed framework have been presented to the industrial companies that have provided the user stories to validate the ideas and to refine the system design by conducting additional focussed interviews.

This work has been conducted within the context of the EU FP7 project LinkedDesign. The rest of the paper is structured as follows: Section 2 provides an overview of Collaborative Planning and identifies the current challenges; Section 3 presents the LinkedDesign project; Section 4 describes the approach that we have taken for our work; Section 5 describes examples of user stories and identifies high-level generic requirements for manufacturing collaboration and IS support; Section 6 presents a framework for collaborative planning, information and decision support system and Section 7 concludes the work and provide future perspectives.

\section{Collaborative Planning}

Collaborative planning is a concept that has been studied by various authors and there is a general notion that collaboration planning has a positive impact on joint decision making in relation to suppliers [6], [7], and new product development [8], forecasting and replenishment [9] and collaborative process planning in product lifecycle management [10]. The suggested benefits of inter and intra-organisational collaborative planning include decreased inventory levels, more predictable order cycle times, elimination of redundant activities, increased product availability and increased sales [11] and [12]. Moreover, effective collaborative planning can be expected to require that the information shared will be of high quality [7].

Many companies rely on technology in the form of collaborative information systems to supplement the traditional modes of communication and information sharing between the customers, suppliers, manufacturers and partners. The main goals of 
these systems are to provide and facilitate the flow of information across and within the organization boundaries while also improving the quality of the shared information. But there is still a lack of visibility and communication within and across the companies. This causes difficulty for the design of interaction mechanisms [13].

Challenges still remain; e.g. existing collaborative planning practices and methodologies have a single organisation's perspective and are restricted to centralised planning and control. There is a lack of flexibility and real time information sharing. The tools do not address the levels of collaboration and compatibility, error handling, engineering changes, data conversion, management of applications, uncertainty and risk adequately. The support available lacks the capability to store and manage the knowledge, experiences and skills; ineffective support for human interaction in distributed teams for efficient decision making and there is a lack of collaborative virtual space for better information sharing and communication.

These challenges pose requirements on the design of IS that support collaborative planning and decision making, e.g.: support for collaboration and coordination, support information dependencies within and between manufacturing enterprises, storage and management of knowledge and skills, support for interoperability to allow free interaction between different entities, support real-time decision making, address errors, operational issues, uncertainties, engineering changes, variations, and risks to cope with the dynamicity and complexity of manufacturing systems, support to improve sourcing; reduce development and operational costs, increase product innovation, and results in a strategic impact on revenue and support for applications management and process alignment.

\section{$3 \quad$ LinkedDesign Project}

LinkedDesign is a European research project (http://www.linkeddesign.eu/) which addresses Linked Knowledge in Manufacturing, Engineering and Design for Next-Generation Production. The central goal of the project is to develop a Linked Engineering and Manufacturing Platform that enables the context-driven and collaborative access to data, information and knowledge from both the engineering and the manufacturing world. The relevant information is scattered in different formats and locations. By using such a platform, engineers will be able to access and integrate (currently disconnected) information sources in a convenient way. To achieve this goal, the project has conducted interviews and workshops with three manufacturing enterprises, the use case providers in the project consortium, to identify their needs.

\section{Approach}

The starting point for this work was the user stories or statements that have been provided by the three industrial partners in the project. The approach that we have taken is illustrated in Fig. 1. In addition to the user stories, we have reviewed relevant literature to identify challenges in collaborative processes. We have described scenarios ("stories about people and their activities" [14]) to obtain a deeper insight into the 
users' needs and to identify the requirements for design and the collaborative processes that involve their work and who they collaborate with. These were used to design a solution.

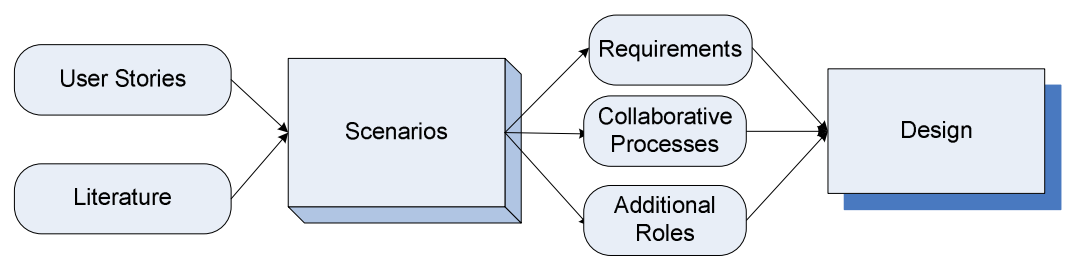

Fig. 1. Design Approach and Process

The design presented in this paper is a refined version of our initial design. The revisions are based on feedback from two industrial partners. Since then, an improved design, presented in this paper, has been presented to the third industrial partner who provided positive feedback

\section{$5 \quad$ User Stories and Requirements}

In this section, we analyse the user stories provided by the industrial partners to obtain high-level requirements to design a collaborative planning, information and decision support system. User stories have been collected from people who fill different roles in manufacturing enterprises, e.g. Design Engineer, IT Specialist, Knowledge Engineer, Project Manager, Supplier, Manufacturer and Customer. These are mostly in the form of statements from specific people expressing a desire or a need for technical support for a specific activity. Examples of user stories or statements from the different roles are provided below.

The Manufacturer, on error handling:

- $\quad$ "I want to be able to retrieve a list of errors that occurred during my shift so that I can evaluate my own performance."

- $\quad$ "I want to see every production error as it occurs so that I may react appropriately."

- $\quad$ "I want to see previous lists of errors so that I can compare parameter settings from production history."

- $\quad$ "I want to see a description of the error type so that I can determine the type of action."

- $\quad$ "I want to be able to access the parameters of the process step immediately before the error occurred."

The Knowledge Engineer on error handling:

- "I want to receive error summaries so that I can analyze possible machine defects."

- $\quad$ "I want to see a detailed history of changes to the system so that I can reevaluate my rule set." 
- $\quad$ "I want be able to formulate rules for error detection so that the system will automatically send error messages."

The Project Manager on error handling:

- $\quad$ I want to receive a report that contains produced parts and number of error parts, monetary equivalent of error parts of the last shift so that I can get an overview of the last shift."

It is important to note that in order to provide access to all relevant information for any user, it requires information that is created by another user or as a result of another user's actions; e.g., the error reports that are desired by the Project Manager may be created during the previous shift by the Manufacturer. Similarly, the error summaries and the detailed history of changes to the system desired by the Knowledge Engineer may be created at another time. Although the user stories are statements from specific people expressing a desire or a need for technical support for a specific activity, it implies collaboration, sometimes implicitly, among several roles; e.g. the Manufacturer and the Knowledge Engineer. It also implies that collaborative planning is central to the activities of the users across the enterprise. The statements also imply that collaboration often occurs through information, e.g. error reports, or perhaps other artifacts or knowledge, e.g. rules.

Timely detection and handling of errors can save time and resources and prevent other problems down the road. The decisions concerning how to deal with errors in collaborative planning, i.e. which strategy to implement, depend on the final goal of the production management and involve many economic and production variables. Also, they depend on the manufacturing type of environment (collaborative, dedicated, flexible, etc.), and the advantages arising from using a certain error handling policy vary from one organisation to another. When an error occurs, the whole or a part of the collaborative plan is concerned. Thus, the development of a preventive off-line planning or that of implementing a real-time decision support system to react to errors is strictly required [15].

An overview of the high-level generic capabilities that were desired by all the users can be summarized as below (this is not an exhaustive list):

- Personalized and role-based support and capabilities.

- Easy access to all relevant information to perform their tasks.

- Access to historical information.

- Real time access to domain knowledge and expertise.

- An overview of all tasks.

- Support for internal knowledge and experience transfer.

- Life cycle assessment.

\section{Collaborative Planning, Information, and Decision Support}

The purpose of this section is to propose a concept of collaborative planning, information and decision support system to carry out collaboration between partners within 
and across organisations and thereby meet the main requirements for IS support identified through the user stories. We have designed some mockups, using the Pencil application, to illustrate some ideas for a collaborative platform to address the needs of the users; see Fig. 2. The main motivation for our design is to bring forth a holistic view and design a collaborative platform to provide support for the different roles in the various processes. One of the main aims of the proposed design is also to support specific processes within an engineering enterprise such as error handling, order management or knowledge management and to provide the various roles involved in these processes personalized, role and task specific support to do their work effectively.

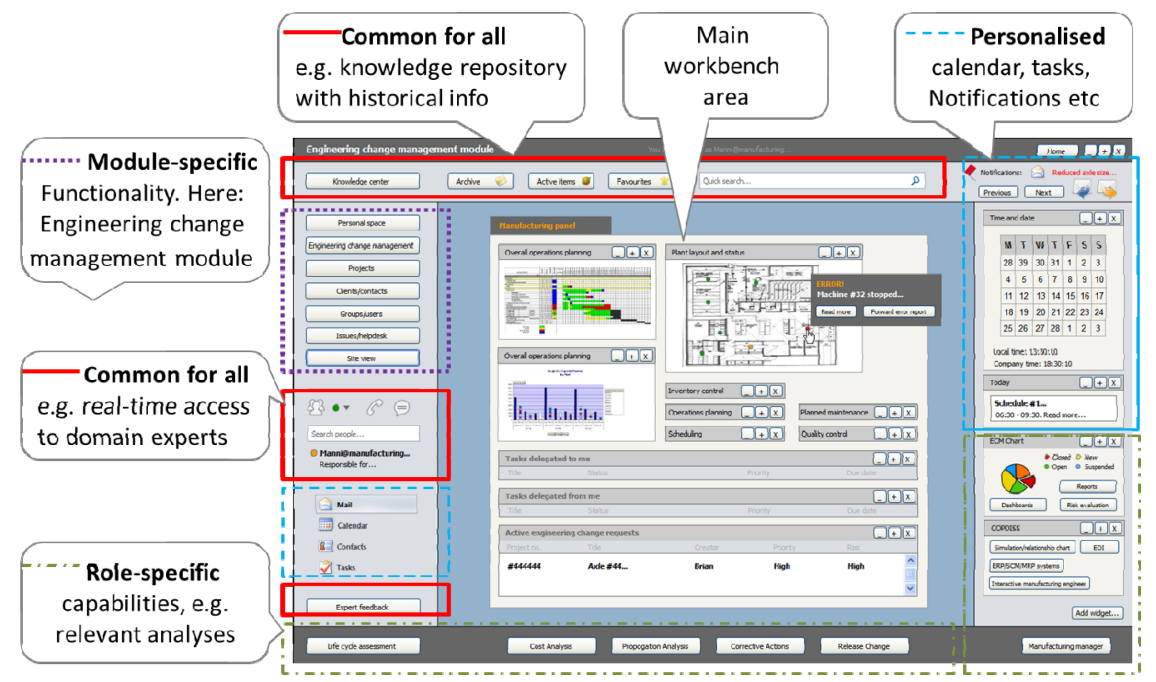

Fig. 2. Design of the functionalities in the Collaborative Platform

To provide personalized or role-based support, it is beneficial for each user to have their own profiles on the platform and some support for their specific as well as general activities. To address the common needs such as the need to access correct and timely information, to have an overview of relevant information and knowledge, both in terms of historical information as well as human expertise, overview of their tasks, we have designed the collaborative platform with some generic functionality that will be accessible for everyone, while executing all processes. The screen layout presented in Fig. 2 is designed to categorize the different types of functionalities that are incorporated into a common workspace for any user. The following categories of functionalities have been considered:

- Common for all: functionality that is available for all roles and individuals. These functionalities are available on the top of the screen, which are dedicated to accessing information sources and searching for information. Another block of common functionalities are placed to the left of the page which provide real time access to other people and can provide an overview of people who are online and available at any time. A similar capability is available to the bottom left of the page where expert feedback is available. 
- Personalized: these functionalities are personalized to the users. Such functionalities include the calendar and task list, available from the top right hand side of the screen and bottom.

- Role-specific: these functionalities are tailored to the role of the person and are framed; e.g. a Design Engineer will see a different set of functionalities compared to a Knowledge Engineer. These are available from the bottom left and the very bottom of the screen.

- Module-specific: these functionalities are tailored to the module or the relevant process, such as error handling or change management, and are placed at the top right and the bottom left of the screen. These functionalities are aimed to support specific processes within enterprises such as handling errors and managing changes during a manufacturing process.

The area in the middle of the screen is dedicated to the role and module specific content. For example, a design engineer will have content that is specific to his/her role and tasks, which is shown on the top right of the area.

\section{$7 \quad$ Summary and Future Work}

In this paper, we have discussed the importance of collaborative planning for manufacturing enterprises. We have analysed user stories and statements provided by industrial partners to understand the collaborative processes in their workplaces and their needs for IS support. Based on the analysis, we have proposed a framework for collaborative planning and ideas for the design of IS support. The design that is presented in this paper is the result of the interviews and feedback on earlier version from the users.

Validation of the mock-up of a collaborative planning, information and decision support system presented in this paper has been conducted with one of the industrial partners. The feedback received was positive and confirmed the need for the types of functionalities proposed in this design. We plan to revisit the other industrial partners in the near future for further validation of the approach and the user interface. Focussed interviews will be conducted to verify the collaborative processes that we have identified through our analyses.

Acknowledgement. This research was done in the LinkedDesign project that has received funding from the European Union's Seventh Framework Programme ([FP7/2007-2013]) under grant agreement no. 284613. We wish to thank the partners for providing the user stories.

\section{References}

1. Lillehagen, F., Krogstie, J.: Active Knowledge Modelling of Enterprises. Springer, Berlin (2008)

2. Bénaben, F., et al.: Collaborative Information System Design. In: AIM 2006 Conference. Lecture Notes in Informatics, pp. 281-296 (2006) 
3. Holweg, M., et al.: Supply Chain Collaboration: Making Sense of the Strategy Continuum. European Management Journal 23, 170-181 (2005)

4. Gonzalez-Ibanez, R., Shah, C.: Coagmento: A system for supporting collaborative information seeking. In: American Society for Information Science and Technology, ASIST 2011, New Orleans, USA (2011)

5. Shafiei, F., Sundaram, D.: Multi-Enterprise collaborative enterprise resource planning and decision support systems. In: 37th Hawaii International Conference on System Sciences, Hawaii, USA (2004)

6. Mohr, J., Spekman, R.: Characteristics of Partnership Success: Partnership Attributes, Communication Behavior, and Conflict Resolution Techniques. Strategic Management Journal 15(2), 135-152 (1994)

7. Monczka, R., et al.: Success Factors in Strategic Supplier Alliances: The Buying Company Perspective. Decision Sciences Journal 29(3), 553-577 (1998)

8. Ragatz, G., Handfield, R., Scannell, T.: Success Factors for Integrating Suppliers into New Product Development. The Journal of Product Innovation Management 29(3), 190-202 (1997)

9. Barratt, M., Oliveira, A.: Exploring the Experiences of Collaborative Planning Initiatives. International Journal of Physical Distribution and Logistics Management 31(4), 266-289 (2001)

10. Ming, X.G., et al.: Collaborative process planning and manufacturing in product lifecycle management. Computers in Industry 59(2-3), 154-166 (2008)

11. Stank, T., Keller, S.B., Daugherty, P.J.: Supply chain collaboration and logistical service performance. Journal of Business Logistics 22(1), 29-48 (2001)

12. Stank, T., Keller, S.B., Arango, M.: Benefits of interfirm coordination in food industry supply chains. Journal of Business Logistics 22(2), 21-41 (1999)

13. Hvolby, H.H., et al.: Collaborative Demand and Supply Planning Networks. In: CruzCunha, M.M., Varajão, J. (eds.) Enterprises Information Systems Design, Implementation and Management: Organizational Applications, pp. 496-504. IGI Global, New York (2011)

14. Carroll, J.M.: Five Reasons for Scenario-Based Design. In: 32nd Hawaii International Conference on System Sciences. IEEE, Hawaii (1999)

15. Bruccoleri, M., Pasek, Z.J., Koren, Y.: Operation management in reconfigurable manufacturing systems: Reconfiguration for error handling. International Journal of Production Economics 100(1), 87-100 (2006) 